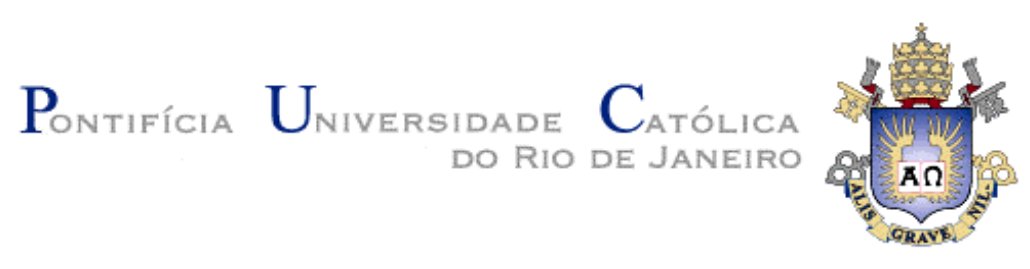

Armando Prestes de Menezes Filho

Não-Extensividade Termodinâmica, Invariância Discreta de Escala e Elasto-Plasticidade: Estudo Numérico de um Modelo Geomecânico Auto-Organizado Criticamente

Tese de Doutorado

Tese apresentada ao Programa de Pós-Graduação em Engenharia Civil da PUC-Rio como requisito parcial para obtenção do título de Doutor em Engenharia Civil. Área de Concentração: Geotecnia.

Orientador: Eurípedes do Amaral Vargas Jr

Rio de Janeiro, 8 de outubro de 2003. 


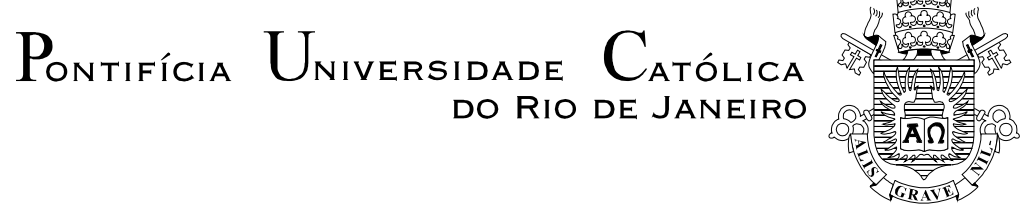

Armando Prestes de Menezes Filho

\section{Não-Extensividade Termodinâmica, Invariância Discreta de Escala e Elasto-Plasticidade: Estudo Numérico de um Modelo Geomecânico Auto-Organizado Criticamente}

Tese apresentada como requisito parcial para a obtenção do título de Doutor pelo Programa de Pós-Graduação em Engenharia Civil do Departamento de Engenharia Civil do Centro Técnico Científico da PUC-Rio. Aprovada pela Comissão Examinadora abaixo assinada.

\section{Prof. Eurípedes do Amaral Vargas Jr Presidente/Orientador Departamento de Engenharia Civil - PUC-Rio \\ Prof. Paulo Batista Gonçalves Departamento de Engenharia Civil - PUC-Rio \\ Profa. Rosane Riera Freire Departamento de Física - PUC-Rio \\ Prof. Raul Rosas e Silva Departamento de Engenharia Civil - PUC-Rio}

Prof. Jorge S. de Sá Martins Instituto de Física - UFF

Prof. Evaldo Mendonça F. Curado Centro Brasileiro de Pesquisas Físicas - CBPF

Prof. Ney Augusto Dumont Coordenador Setorial Do Centro Técnico Científico - PUC-Rio

Rio de Janeiro, 8 de outubro de 2003. 
Todos os direitos reservados. É proibida a reprodução total ou parcial do trabalho sem autorização da universidade, do autor e do orientador.

\section{Armando Prestes de Menezes Filho}

Graduou-se em Engenharia Civil pela UFRJ em 1982, e obteve o mestrado pela PUC-Rio em 1993. Foi agraciado com o Prêmio Icarahy da Silveira da ABMS. Bolsista de doutorado pelo CNPq junto ao Departamento de Física da Universidade de Oslo, Noruega. Pesquisador no Norwegian Geotechnical Institute (NGI) e no Institute for Energy Technology (IFE). Foi professor dos Departamentos de Engenharia Civil da PUC-Rio, de Geologia do IGEO/ UFRJ e de Engenharia Civil da UENF.

Ficha Catalográfica

Prestes de Menezes Filho, Armando

Não-extensividade termodinâmica, invariância discreta de escala e elasto-plasticidade: estudo numérico de um modelo geomecânico auto-organizado criticamente / Armando Prestes de Menezes Filho; orientador: Eurípedes Vargas do Amaral Jr. - Rio de Janeiro: PUC, Departamento de Engenharia Civil, 2003.

v., 189f.: il. ; $29,7 \mathrm{~cm}$.

Tese (doutorado) - Pontifícia Universidade Católica do Rio de Janeiro, Departamento de Engenharia Civil.

Inclui referências bibliográficas.

1.Engenharia civil - Teses. 2.Criticalidade auto-organizada. 3.Invariância discreta de escala. 4.Entropia de Tsallis. 5.Material elasto-plástico. 6. Ruptura progressiva I. Vargas, Eurípedes do Amaral. II. Pontifícia Universidade Católica do Rio de Janeiro. Departamento de Engenharia Civil. III. Título. 
Assim termina a história de uma viagem, que vocês viram e ouviram:

e viram o que é comum, o que está sempre ocorrendo.

Mas a vocês nós pedimos: no que não é de estranhar, procurem o que há de estranho!

No que parece normal, vejam o que há de anormal!

No que parece explicado, vejam o quanto não se explica!

E o que parece comum, vejam como é de espantar! Na regra, vejam o abuso! E onde o abuso apontar, procurem remediar!

(Bertolt Brecht, A Exceção e a Regra) 


\section{Agradecimentos}

$\mathrm{O}$ autor gostaria de deixar consignado seu profundo agradecimento às pessoas e instituições, que colaboraram para a consecução desta pesquisa, resultado de um trabalho desenvolvido em dois países, Brasil e Noruega:

No Institute for Energy Technology (IFE), agradeço ao pesquisador Jiri Muller, a orientação e estímulo proporcionados durante a primeira fase deste trabalho. Ao Prof. Arne T. Skjeltorp, diretor do departamento de física do instituto, que, do alto de sua grande experiência e humildade, soube me orientar no sentido da importância dos sistemas complexos e dos fenômenos críticos para as geociências. Sou grato também ao Prof. Terie Syra, Antoine Saucier, Sigmund Claussen, Olaf Huseby, e, especialmente, à Cristina e Cláudio Lattini, pela amizade sincera, que tornaram a adaptação minha e de minha família muito mais fácil.

No Norwegian Geotechnical Institute (NGI), agradeço ao Prof. Kaare Hoeg, pela atenção e estímulo recebidos durante minha estadia como pesquisador daquele renomado instituto. Ao pesquisador Marte Gutierrez, entusiasta dos fractais, que sempre me apoiou e ajudou. Agradeço, também, a Ramón Verdugo, o chileno de alma mais brasileira que conheço, pela amizade construída durante sua passagem como pesquisador pós-doc daquele instituto.

No Departamento de Física da Universidade de Oslo, sou grato aos Profs. Jens Feder e T. Jossang por me permitirem acompanhar os cursos sobre fenômenos críticos, especialmente lecionados em inglês, e a Jens Lothe, particularmente pelo seu exemplo de extrema modéstia. Agradeço, igualmente, aos Profs. Amnon Aharony, Paul Meakin e Alex Hansen por haverem me apresentado ao maravilhoso mundo dos sistemas complexos, fractais e caos.

No Departamento de Engenharia Civil da PUC-RIO, sou grato, muito 
especialmente, ao Prof. Eurípedes Vargas, pela orientação na segunda etapa desta pesquisa, pelo seu exemplo de modéstia, sempre disposto a aprender e a estudar coisas novas, tendo sempre se mostrado interessado e me estimulado a ir adiante.

Agradeço, igualmente, ao Prof. Rodrigo Figueiredo, da Escola de Minas de Ouro Preto, pelas orientações a respeito da relaxação dinâmica.

Aos Profs. Constantino Tsallis e Evaldo Curado, do Centro Brasileiro de Pesquisas Físicas (CBPF), pela troca de idéias extremamente estimulante, e valiosas sugestões sobre a mecânica estatística generalizada e a não-extensividade termodinâmica.

Aos meus queridos amigos Sergio Tibana, Ricardo Rovo e Peter Fleming, pelo muito que me ajudaram, pelo apoio e atenção recebidos.

A todos os colegas e funcionários da PUC-Rio pela convivência.

À PUC-Rio e ao CNPq pelo auxílio financeiro, sem o qual a realização desta pesquisa teria sido impossível.

À minha mãe, que muito me ajudou, sabendo suportar a minha ausência por um longo par de anos. Agradeço, também, à minha irmã, que muito me apoiou.

Por fim, um agradecimento muitíssimo especial é dirigido à minha querida família, verdadeira e genuinamente, a minha melhor parte. À minha querida e muito amada esposa Geórgia e às minhas filhas Renata e Patrícia, pelo apoio incondicional, carinho, amor e solidariedade nos bons e maus momentos, vividos em dois países tão diferentes. Agradeço muito a Deus e à vida por me haver permitido fazer parte de seus universos. É a estas três jóias raras que dedico este trabalho. 


\section{Resumo}

Prestes de Menezes Filho, Armando; Vargas, Eurípedes do Amaral. NãoExtensividade Termodinâmica, Invariância Discreta de Escala e ElastoPlasticidade: Estudo Numérico de um Modelo Geomecânico AutoOrganizado Criticamente. Rio de Janeiro, 2003. 189p. Tese de Doutorado Departamento de Engenharia Civil, Pontifícia Universidade Católica do Rio de Janeiro.

Esta tese busca utilizar os novos conceitos físicos relacionados à física do estado sólido e à mecânica estatística - teoria do caos e geometria fractal - na análise do comportamento de sistemas dinâmicos não-lineares.

Mais pormenorizadamente, trata-se de estudar o comportamento de um modelo numérico elasto-plástico com função de escoamento de Mohr-Coulomb, usualmente empregado em simulações de materiais geológicos - cimentados ou não -, quando submetido a carregamentos externos, situação esta geralmente encontrada em problemas afeitos à mecânica dos solos e das rochas (p/ex., estabilidade de taludes e escavações subterrâneas).

Mostra-se que tal modelo geomecânico de muitos corpos (many-body) interagentes é conduzido espontaneamente, ao longo de sua evolução temporal, à chamada criticalidade auto-organizada (self-organized criticality - SOC), estado caracterizado por apresentar evolução na fronteira entre ordem e caos, sensibilidade extrema a qualquer pequena perturbação, e desenvolvimento de interações espaço-temporais de longo alcance.

Como a evolução de qualquer sistema dinâmico pode ser vista como um fluxo ininterrupto de informações entre suas partes constituintes, avaliou-se, para tal sistema, a entropia de Tsallis, formulação original proposta pelo físico brasileiro Constantino Tsallis, do Centro Brasileiro de Pesquisas Físicas (CBPF), tendo se mostrado adequada à sua descrição.

Em especial, determinou-se para tal sistema, pela primeira vez, o valor do índice entrópico, que parametriza a aludida forma entrópica alternativa.

Ademais, como é característico de sistemas fora do equilíbrio regidos por 
uma dinâmica de limiar, mostra-se que tal sistema geomecânico, durante o seu desenvolvimento, teve a sua simetria translacional inicial quebrada, sendo substituída pela simetria por escala, auto-semelhante (i.é., fractal). Em decorrência, o modelo exibe a chamada invariância discreta de escala (discrete scale invariance - DSI), fruto do processo mesmo de ruptura progressiva do material heterogêneo.

Especificamente, as simulações numéricas sugeriram que o processo de ruptura progressiva do material elasto-plástico se dá por uma transferência multiplicativa de tensões, em diferentes escalas de observação hierarquicamente dispostas, acarretando o aparecimento de sinais bastante peculiares, caracterizados por desvios oscilatórios sistemáticos do padrão em lei de potência, o que possibilita a previsão de sua ruína, quando ainda em fase preparatória.

Assim, esta pesquisa mostrou a eficiência de tal método de previsão, aplicado, pela primeira vez, não somente aos resultados das simulações numéricas do referido modelo geomecânico, como aos ensaios de laboratório em rochas sedimentares, realizados no Centro de Pesquisas da Petrobrás (CENPES).

Por fim, é interessante assinalar que o material elasto-plástico investigado neste trabalho teve seu comportamento compartilhado por um modelo matemático bastante simples, fundamentado na função binomial multifractal, reconhecida por descrever processos multiplicativos em diferentes escalas.

\section{Palavras-chave}

Criticalidade Auto-Organizada; Invariância Discreta de Escala; Entropia de Tsallis; Material Elasto-Plástico; Ruptura Progressiva. 


\section{Abstract}

Prestes de Menezes Filho, Armando; Vargas, Eurípedes do Amaral. Thermodynamic Nonextensivity, Discrete Scale Invariance and Elastoplasticity: A Study of a Self-Organized Critical Geomechanical Numerical Model. Rio de Janeiro, 2003. 189p. DSc Thesis - Department of Civil Engineering, Pontifícia Universidade Católica do Rio de Janeiro.

This thesis aims at applying new concepts from solid state physics and statistical mechanics - chaos theory and fractal geometry - to the study of nonlinear dynamic systems.

More precisely, it deals with a two-dimensional continuum elastoplastic Mohr-Coulomb model, commonly used to simulate pressure-sensitive materials (e.g., soils, rocks and concrete) subjected to stress-strain fields, normally found in general soil or rock mechanics problems (e.g., slope stability and underground excavations).

It is shown that such many-body system is spontaneously driven to a state at the edge of chaos, called self-organized criticality (SOC), capable of developing long-range interactions in space and long-range memory in time.

A new entropic form proposed by $\mathrm{C}$. Tsallis is presented and shown that it is the suitable theoretical framework to deal with these problems.

Furthermore, the index q of the Tsallis entropy, which measures the degree of non-additivity of the system, is calculated, for the first time, for an elastoplastic model.

In addition, as is usual in non-equilibrium systems with threshold dynamics, the model changes its symmetry, from translational to fractal (that is, self-similar), leading to what is called discrete scale invariance.

It is shown that this special type of scale invariance, characterized by systematic oscillatory deviations from the fundamental power-law behavior, can be used to predict the failure of heterogeneous materials, while the process is still being build-up, i.e., from precursory signals, typical of progressive failure processes. 
Specifically, this framework was applied, for the first time, not only to the elastoplastic geomechanical model, but to laboratory tests in sedimentary rocks as well.

Finally, it is interesting to realize that the above-mentioned behaviors are also displayed by the binomial multifractal function, known to adequately describe multiplicative cascading processes.

\section{Keywords}

Self-Organized Criticality; Discrete Scale Invariance; Tsallis Entropy; Elastoplastic Model; Progressive Failure. 


\section{Sumário}

1 Apresentação 22

1.1. Introdução 22

1.2. Caos e fractais: conceitos fundamentais 27

2 Sistemas complexos $\quad 36$

2.1. Introdução 36

2.2. O que são sistemas complexos? 36

2.3. Características dos sistemas complexos 38

2.4. Os sistemas complexos e as geociências 41

3 Não-extensividade termodinâmica 46

3.1 Introdução 46

3.2 Limitações da mecânica estatística e termodinâmica tradicionais $\quad 47$

3.3 Formalismo tradicional aplicado à difusão anômala unidimensional 54

3.4 Breve exposição da não-extensividade entrópica 56

3.5 Quantificação da não-extensividade 59

4 Invariância discreta de escala $\quad 67$

$\begin{array}{ll}4.1 \text { Introdução } & 67\end{array}$

4.2 Invariâncias de escala contínua e discreta, e sua formulação

matemática

68

4.3 Aspectos preditivos das leis de potência pura e corrigida log-

periodicamente

72

4.4 Um exemplo de processo em cascata: a função binomial multifractal77

4.5 Processo em cascata e a entropia de Tsallis

4.6 Processo em cascata e as invariâncias contínua e discreta de escala91

5 Descrição do modelo geomecânico elasto-plástico 99

5.1 Introdução 99

5.2 Formulação matemática do modelo elasto-plástico 99 
5.3 Forma variacional das equações de campo

103

5.4 Relaxação dinâmica 106

5.5 Procedimento de integração numérica 108

5.6 Parâmetros e condições de contorno adotados nas simulações numéricas 108

5.6.1 Para as simulações dos capítulos 6 e $7 \quad 109$

5.6.2 Para as simulações do capítulo 8

6 Elasto-plasticidade e criticalidade auto-organizada Resultados experimentais 113

6.1 Introdução 113

6.2 Criticalidade auto-organizada e modelos geomecânicos 113

6.3 Primeiro sinal de SOC: desenvolvimento de interações temporais de longo alcance 116

6.4 Segundo sinal de SOC: desenvolvimento de interações espaciais de longo alcance 125

7 Elasto-plasticidade e não-extensividade termodinâmica Resultados experimentais 135

7.1 Introdução 135

7.2 Determinação do parâmetro não-extensivo pelo procedimento (A) 136

$\begin{array}{ll}7.3 \text { Resultados experimentais } & 137\end{array}$

8 Elasto-plasticidade e invariância discreta de escala Resultados experimentais 143

8.1 Introdução 143

8.2 Descrição do comportamento do modelo elastoplástico pela invariância discreta de escala

8.3 Previsões proporcionadas pelas invariâncias contínua e discreta de escala

8.3.1 Resultados provenientes das análises numéricas realizadas no modelo geomecânico

8.3.2 Resultados provenientes de ensaios realizados em laboratório $\quad 157$

8.3.2.1 Verificação da invariância contínua de escala (expressão 4.18) 160 
8.3.2.2 Verificação da invariância discreta de escala (expressão 4.17) 163

9 Considerações finais 166

9.1 Conclusões 166

9.2 Sugestões para futuras pesquisas 169

10 Referências bibliográficas 172 


\section{Lista de figuras}

Figura 1.1 - Triângulo de Sierpinski. 28

Figura 1.2 - Curva de von Koch. 28

Figura 1.3 - Agregado fractal limitado por difusão. 29

Figura 1.4 - Espectro de dimensões generalizadas. 33

Figura 1.5 - Espectro de singularidades. 33

Figura 1.6 - Atrator de Lorenz. 34

Figura 3.1 - Cascata de bifurcação de Feigenbaum. 60

Figura 3.2 - Evolução temporal da função de sensibilidade, para o expoente de Lyapunov inferior a zero. A curva cheia representa uma convergência exponencial (gráfico log-log). 61

Figura 3.3 - Evolução temporal da função de sensibilidade, para o expoente de Lyapunov superior a zero. A curva cheia representa uma divergência exponencial (gráfico log-log).

Figura 3.4 - Variação do expoente de Lyapunov com o parâmetro de controle, para o mapa logístico.

Figura 3.5 - Evolução temporal da função de sensibilidade, para o valor de a crítico. Notar o aspecto fractal da curva, que apresenta uma divergência em lei de potência.

Figura 4.1 - Esquema multiplicativo da função binomial multifractal (fractal de de Wijs). Os números que aparecem entre parênteses referem-se aos diferentes níveis hierárquicos em que a massa é distribuída [106].

Figura 4.2 - Processo multiplicativo representado por uma árvore fractal.

Como exemplo, a distribuição de uma força $\mathrm{F}$ por seus diversos níveis hierárquicos [96]. 78

Figura 4.3 - Função binomial multifractal não-acumulada $(b=0.10) . \quad 79$

Figura 4.4 - Função binomial multifractal acumulada $(b=0.10)$.

Figura 4.5 - Função binomial multifractal não-acumulada $(b=0.25)$. Esta figura deve ser comparada com a figura 3.5.

Figura 4.6 - Função binomial multifractal acumulada $(b=0.25)$.

Figura 4.7 - Função binomial multifractal não-acumulada $(b=0.40) . \quad 81$ 
Figura 4.8 - Função binomial multifractal acumulada $(b=0.40)$.

Figura 4.9 - Função binomial multifractal acumulada $(b=0.50)$.

Figura 4.10 - Função binomial multifractal não-acumulada $(b=0.70) . \quad 82$

Figura 4.11 - Função binomial multifractal acumulada $(b=0.70)$.

Figura 4.12 - Função binomial multifractal não-acumulada $(b=0.85)$. 83

Figura 4.13 - Função binomial multifractal acumulada $(b=0.85)$.

Figura 4.14 - Espectro de dimensões generalizadas da função binomial multifractal $(b=0.10)$. Observar que, no extremo inferior direito, as dimensões são muito próximas a zero.

Figura 4.15 - Espectro de dimensões generalizadas da função binomial multifractal $(b=0.25)$. Notar a magnitude dos valores situados no eixo das ordenadas.

Figura 4.16 - Espectro de dimensões generalizadas da função binomial multifractal $(b=0.495)$. Destruição gradual da multifractalidade (observar a magnitude dos valores do eixo das ordenadas).

Figura 4.17 - Espectro de singularidade da função binomial multifractal ( $b$ = 0.10). Notar o alfa mínimo próximo a zero.

Figura 4.18 - Espectro de singularidade da função binomial multifractal ( $b$ $=0.25)$.

Figura 4.19 - Espectro de singularidade da função binomial multifractal ( $b$ = 0.495). Notar a aproximação dos alfas máximo e mínimo, e a consequente destruição paulatina da multifractalidade.

Figura 4.20 - Variação do índice entrópico de Tsallis com o parâmetro $b$. Observar que, para uma distribuição eqüitativa de masa $(b=0.50)$, o índice tende para a infinitude negativamente. Por outro lado, para distribuições de massa cada vez mais heterogêneas, o índice se aproxima da unidade.

Figura 4.21 - Ajuste da função binomial $(b=0.10)$ pela lei de potência pura (expressão 4.18).

Figura 4.22 - Ajuste da função binomial $(b=0.25)$ pela lei de potência pura (expressão 4.18).

Figura 4.23 - Ajuste da função binomial $(b=0.40)$ pela lei de potência pura (expressão 4.18). 
Figura 4.24 - Ajuste da função binomial $(b=0.10)$ pela lei log-periódica (expressão 4.17).

Figura 4.25 - Ajuste da função binomial $(b=0.25)$ pela lei log-periódica (expressão 4.17).

Figura 4.26 - Ajuste da função binomial $(b=0.40)$ pela lei log-periódica (expressão 4.17).

Figura 5.1 - Malha de elementos finitos (400 elementos e 221 nós). 109

Figura 6.1 - Atrator do sistema elasto-plástico amortecido.

Figura 6.2 - Evolução temporal (número de iterações) da deformação plástica equivalente média (CC1, MPP). O coeficiente de amortecimento é igual a 0.001.

Figura 6.3 - Evolução temporal (número de iterações) da deformação plástica equivalente média (CC1, MPP). O coeficiente de amortecimento é igual a 0.01 .

Figura 6.4 - Evolução temporal (número de iterações) da deformação plástica equivalente média (CC1, MPA). O coeficiente de amortecimento é igual a 0.001 .

Figura 6.5 - Evolução temporal (número de iterações) da deformação plástica equivalente média (CC2, MPP). O coeficiente de amortecimento é igual a 0.001 .

Figura 6.6 - Evolução temporal (número de iterações) da deformação plástica equivalente média (CC2, MPP). O coeficiente de amortecimento é igual a 0.01 .

Figura 6.7 - Evolução temporal (número de iterações) da deformação plástica equivalente média (CC3, MPP). O coeficiente de amortecimento é igual a 0.001. A linha tracejada representa uma evolução em lei exponencial (observar a escala de valores no eixo das ordenadas). 120 Figura 6.8 - Expoente de Lyapunov efetivo calculado da série temporal da figura 6.2 .

Figura 6.9 - Expoente de Lyapunov efetivo calculado da série temporal da figura 6.3.

Figura 6.10 - Expoente de Lyapunov efetivo calculado da série temporal da figura 6.4 . 
Figura 6.11 - Expoente de Lyapunov efetivo calculado da série temporal da figura 6.5.

Figura 6.12 - Expoente de Lyapunov efetivo calculado da série temporal da figura 6.6.

Figura 6.13 - Expoente de Lyapunov efetivo calculado da série temporal da figura 6.7 .

Figura 6.14 - Isocurvas de deformação plástica equivalente, mostrandose as bandas de cisalhamento (área hachurada). $\mathrm{O}$ coeficiente de amortecimento é igual a 0.001 (CC1, MPP).

Figura 6.15 - Isocurvas de deformação plástica equivalente, mostrandose as bandas de cisalhamento. Notar as distorções na área hachurada, fruto do super-amortecimento. $O$ coeficiente de amortecimento é igual a 0.01 (CC1, MPP).

Figura 6.16 - Isocurvas de deformação plástica equivalente, mostrandose as bandas de cisalhamento (área hachurada). O coeficiente de amortecimento é igual a 0.001 (CC1, MPA).

Figura 6.17 - Isocurvas de deformação plástica equivalente, mostrandose as bandas de cisalhamento (área hachurada). $\mathrm{O}$ coeficiente de amortecimento é igual a 0.001 (CC2, MPP).

Figura 6.18 - Isocurvas de deformação plástica equivalente. Observar a ausência de padrão espacial, comparativamente às figuras anteriores. $O$ coeficiente de amortecimento é igual a 0.001 (CC3, MPP).

Figura 6.19 - Determinação da dimensão fractal $D_{0}=1.79$ (expressão 1.1) das bandas de cisalhamento (área hachurada) da figura 6.14 (CC1, MPP).1 Figura 6.20 - Determinação da dimensão de correlação $D_{2}=1.62$ (expressão 1.12) das bandas de cisalhamento (área hachurada) da figura 6.14 (CC1, MPP).

Figura 6.21 - Determinação da dimensão fractal $D_{0}=1.79$ (expressão

1.1) das bandas de cisalhamento (área hachurada) da figura 6.16 (CC1, MPA).

Figura 6.22 - Determinação da dimensão de correlação $D_{2}=1.65$

(expressão 1.12) das bandas de cisalhamento (área hachurada) da figura 6.16 (CC1, MPA). 
Figura 6.23 - Determinação da dimensão fractal $D_{0}=1.79$ (expressão 1.1) das bandas de cisalhamento (área hachurada) da figura 6.17 (CC2, MPP).

Figura 6.24 - Determinação da dimensão de correlação $D_{2}=1.61$

(expressão 1.12) das bandas de cisalhamento (área hachurada) da figura

6.17 (CC2, MPP).

Figura 6.25 - Isocurvas de deformação plástica equivalente, mostrandose as bandas de cisalhamento (área hachurada). O número de elementos da malha é de 3200 , sendo o coeficiente de amortecimento igual a 0.001 (CC1, MPP).

Figura 6.26 - Determinação da dimensão fractal $D_{0}=1.11$ (expressão 1.1) das bandas de cisalhamento (área hachurada) da figura 6.25, para o caso de uma malha de 3200 elementos (CC1, MPP).

Figura 7.1 - Evolução temporal coletiva t do dano normalizado $<\mathrm{D}(\mathrm{t})>\mid<\mathrm{D}(1)>$ (média de 4 (quatro) realizações). A reta de inclinação $\delta=$ $3.497(q=0.71)$ é um guia para os olhos. O coeficiente de amortecimento é igual a 0.001 (CC1, MPP).

Figura 7.2 - Evolução temporal coletiva t do dano normalizado $<\mathrm{D}(\mathrm{t})>\mid<\mathrm{D}(1)>$ (média de 4 (quatro) realizações). A reta de inclinação $\delta=$ 3.217 ( $q=0.69$ ) é um guia para os olhos. $O$ coeficiente de amortecimento é igual a 0.01 (CC1, MPP).

Figura 7.3 - Evolução temporal coletiva t do dano normalizado $<\mathrm{D}(\mathrm{t})>\mid<\mathrm{D}(1)>$ (média de 4 (quatro) realizações). A reta de inclinação $\delta=$ $3.295(q=0.70)$ é um guia para os olhos. O coeficiente de amortecimento é igual a 0.001 (CC1, MPA).

Figura 7.4 - Evolução temporal coletiva t do dano normalizado $\langle\mathrm{D}(\mathrm{t})>|<\mathrm{D}(1)>$ (média de 4 (quatro) realizações). A reta de inclinação $\delta=$ $3.366(q=0.70)$ é um guia para os olhos. O coeficiente de amortecimento é igual a 0.001 (CC2, MPP).

Figura 7.5 - Evolução temporal coletiva t do dano normalizado $\langle\mathrm{D}(\mathrm{t})>|<\mathrm{D}(1)>$ (média de 4 (quatro) realizações). A reta de inclinação $\delta=$ $3.234(q=0.69)$ é um guia para os olhos. $O$ coeficiente de amortecimento é igual a 0.01 (CC2, MPP). 
Figura 7.6 - Evolução temporal coletiva t do dano normalizado $<\mathrm{D}(\mathrm{t})>\mid<\mathrm{D}(1)>$ (média de 4 (quatro) realizações). A linha cheia de inclinação $\delta=8.590(q=0.88)$ é uma lei de potência, ao passo que a tracejada é uma exponencial (gráfico semi-log). O coeficiente de amortecimento é igual a 0.001 (CC3, MPP). 140

Figura 8.1 - Energia cinética desenvolvida no nó 17.

Figura 8.2 - Energia cinética acumulada no nó 17.

Figura 8.3 - Distribuição estatística em lei de potência da energia cinética para o nó 17. A inclinação da reta tracejada é igual a 2.39. 146

Figura 8.4 - Energia cinética no nó 17. Janela de tempo escolhida para estudo.

Figura 8.5 - Energia cinética acumulada no nó 17. Janela temporal escolhida para estudo

Figura 8.6 - Energia cinética acumulada no nó 17. Ajuste em lei de potência (expressão 4.18).

Figura 8.7 - Energia cinética acumulada no nó 17. Ajuste pela lei logperiódica (expressão 4.17).

Figura 8.8 - Energia cinética acumulada no nó 17. Ajuste pela lei logperiódica (expressão 4.17). Desta feita, o tempo crítico é um parâmetro de ajuste.

Figura 8.9 - Energia cinética desenvolvida no nó 17. Janela de tempo escolhida para estudo.

Figura 8.10 - Energia cinética acumulada no nó 17. Ajuste pela lei logperiódica (expressão 4.17). O tempo crítico é um parâmetro de ajuste. 155 Figura 8.11 - Curva tensão - deformação do ensaio de compressão uniaxial em folhelho.

Figura 8.12 - Evolução temporal do deslocamento, medido pelo LVDT, no ensaio de compressão uniaxial em folhelho.

Figura 8.13 - Evolução temporal do deslocamento, medido pelo LVDT, no ensaio de compressão uniaxial em folhelho (dados incompletos). 160

Figura 8.14 - Ajuste em lei de potência (expressão 4.18) aos dados de deslocamento da figura 8.13.

Figura 8.15 - Extensão do ajuste pela expressão (4.18) à totalidade dos 
dados experimentais.

Figura 8.16 - Ajuste pela lei log-periódica (expressão 4.17) aos dados de deslocamento da figura 8.13.

163

Figura 8.17 - Extensão do ajuste pela expressão (4.17) à totalidade dos dados experimentais. 


\section{Lista de tabelas}

Tabela 4.1 - Dados referentes à função binomial multifractal. $\quad 89$ Tabela 4.2 - Dados relativos ao ajuste em lei de potência pura (expressão 4.18).

Tabela 4.3 - Dados relativos ao ajuste segundo a lei log-periódica (expressão 4.17).

Tabela 7.1 - Valores do índice entrópico de Tsallis estimado pela sensibilidade do sistema às condições iniciais ("mixing"). 